\title{
Isolable Diphosphorus-Centered Radical Anion and Diradical Dianion
}

Gengwen Tan, $\uparrow$ Shuyu Li, $\uparrow$ Sheng Chen, $\uparrow$ Yunxia Sui,,$\uparrow$ Yue Zhao, $\uparrow$ Xinping Wang $* \dagger$

$\dagger$ State Key Laboratory of Coordination Chemistry, School of Chemistry and Chemical Engineering, Nanjing National Laboratory of Microstructures, Nanjing University, Nanjing 210093, P.R. China $\$$ Center of Modern Analysis, Nanjing University, Nanjing 210093, P.R. China

E-Mail: xpwang@nju.edu.cn

\section{Contents}

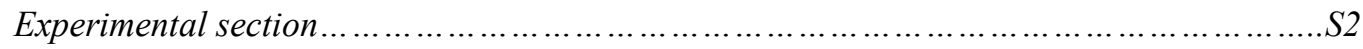

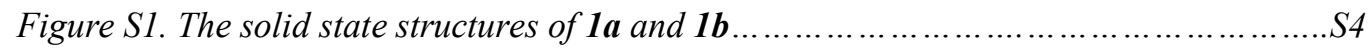

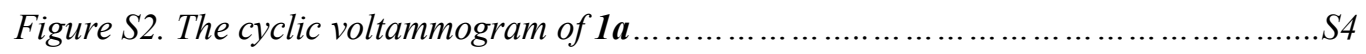

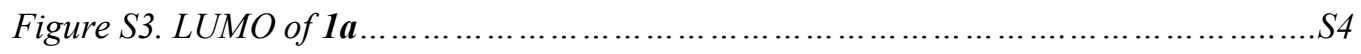

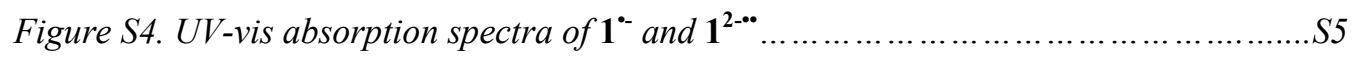

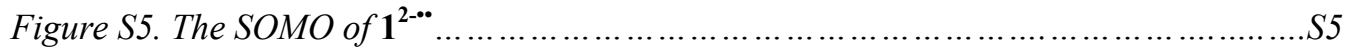

Table S1. Crystal Data and Structure Refinement .............................................. 6

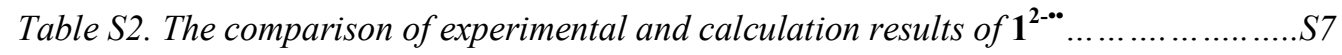

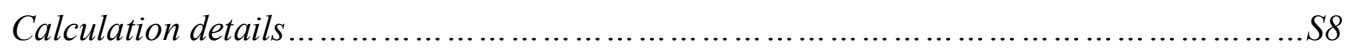

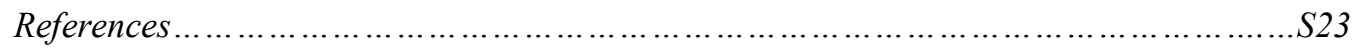




\section{Experimental section}

General considerations: All experiments were carried out under a dry oxygen-free nitrogen atmosphere using standard Schlenk techniques. Solvents were dried by standard methods and freshly distilled prior to use. The NMR spectra were recorded on Bruker spectrometers (AV400 and AV300) referenced to residual solvent signals as internal standards $\left({ }^{1} \mathrm{H}\right.$ NMR: $\mathrm{CDCl}_{3}, 7.26 \mathrm{ppm}$ and ${ }^{13} \mathrm{C}\left\{{ }^{1} \mathrm{H}\right\}$ NMR: $\left.\mathrm{CDCl}_{3}, 77.2 \mathrm{ppm}\right)$ or with an external standard $\left(\mathrm{H}_{3} \mathrm{PO}_{4}\right.$ for ${ }^{31} \mathrm{P}\left\{{ }^{1} \mathrm{H}\right\}$ NMR). Abbreviations: $\mathrm{s}=$ singlet; $\mathrm{m}=$ multiplet. Cyclic voltammetry was performed on a CHI660E electrochemical workstation with platinum as the working electrode and $\mathrm{Ag} / \mathrm{AgNO}_{3}\left(0.1 \mathrm{M}\right.$ in $\left.\mathrm{CH}_{3} \mathrm{CN}\right)$ as the reference, and the measurement was carried out under a nitrogen atmosphere. EPR spectra were obtained using Bruker EMX plus-6/1 X-band variable-temperature apparatus. UV-vis spectra were recorded on Lambda 750 spectrometer. Element analyses were performed at Shanghai Institute of Organic Chemistry, the Chinese Academy of Sciences. For the single crystal X-ray structure analyses the crystals were each mounted on a glass capillary in perfluorinated oil and measured in a cold $\mathrm{N}_{2}$ flow. The data were collected on Bruker D8 CMOS detectors at $123 \mathrm{~K}$. The structures were solved by direct methods and all refined on $F^{2}$ with the SHELX-97 software package. The positions of the $\mathrm{H}$ atoms were calculated and considered isotropically according to a riding model. Commercially available reagents were purchased from Aldrich, Acros or Alfa-Assar, and used as received. $\mathrm{Mes}^{*} \mathrm{PCl}_{2}{ }^{1}$ and indofluorene ${ }^{2}$ were synthesized according to the reported procedures.

Synthesis of the diphosphaalkenes $\mathbf{1 a}$ and $\mathbf{1 b}$ : The suspension of indofluorene $(0.881 \mathrm{~g}, 3.46$ $\mathrm{mmol}$ ) in THF was cooled to $-78{ }^{\circ} \mathrm{C}$ and $n \mathrm{BuLi}(3.04 \mathrm{~mL}, 2.5 \mathrm{M}$ in $n$-hexane, $7.60 \mathrm{mmol})$ was added dropwise, then the mixture was allowed to slowly warm up to room temperature under stirring. After stirring for 2 hours, the dark green suspension was transferred to a flask containing $\mathrm{Mes}^{*} \mathrm{PCl}_{2}(2.805 \mathrm{~g}, 8.08 \mathrm{mmol})$ in THF $(10 \mathrm{~mL})$ at $-78{ }^{\circ} \mathrm{C}$ via cannula under vigorous stirring. The mixture was stirred at this temperature for 1 hour, and then left to stir at room temperature for another 2 hours to afford a brown-yellow solution. An excess amount of 1,8-diazabicyclo[5.4.0]undec-7-ene (DBU) $(1.37 \mathrm{~mL}, 9.17 \mathrm{mmol})$ was added at room temperature, and the mixture was stirred at room temperature for 48 hours to give a deep orange suspension. All the volatiles were removed in vacuo, and the residue was purified by column chromatography (silica) with petroleum ether $/ \mathrm{CH}_{2} \mathrm{Cl}_{2}(\mathrm{v} / \mathrm{v} 20: 1)$ as the eluent, and compounds 1a ( $0.49 \mathrm{~g}, 0.59 \mathrm{mmol}, 17 \%)$ and $\mathbf{1 b}(0.12 \mathrm{~g}, 0.14 \mathrm{mmol}, 4 \%)$ were isolated after column chromatography as a red solid. NMR data for 1a: ${ }^{1} \mathrm{H} \mathrm{NMR}\left(\mathrm{CDCl}_{3}, 400 \mathrm{MHz}, 298 \mathrm{~K}\right)$ : $\delta(\mathrm{ppm})=1.42\left(\mathrm{~s}, 18 \mathrm{H},\left(\mathrm{CH}_{3}\right)_{3} \mathrm{C}\right), 1.45\left(\mathrm{~s}, 9 \mathrm{H},\left(\mathrm{CH}_{3}\right)_{3} \mathrm{C}\right), 1.48\left(\mathrm{~s}, 18 \mathrm{H},\left(\mathrm{CH}_{3}\right)_{3} \mathrm{C}\right), 1.51(\mathrm{~s}, 9$ $\left.\mathrm{H},\left(\mathrm{CH}_{3}\right)_{3} \mathrm{C}\right), 4.98\left(\mathrm{~d}, 1 \mathrm{H},{ }^{3} J(\mathrm{H}, \mathrm{H})=8.1 \mathrm{~Hz}, \mathrm{Ar}-H\right), 6.00(\mathrm{~s}, 1 \mathrm{H}, \mathrm{Ar}-H), 6.58-6.63(\mathrm{~m}, 1 \mathrm{H}$, Ar-H), 6.93-7.00 (m, 2 H, Ar-H), 7.28-7.32 (m, 1 H, Ar-H), 7.36-7.39 (m, 1 H, Ar- $H$ ), 7.53 (s, $\left.2 \mathrm{H}, \mathrm{Mes}^{*}-H\right), 7.65$ (s, $\left.2 \mathrm{H}, \mathrm{Mes}^{*}-H\right), 7.75\left(\mathrm{~d}, 1 \mathrm{H},{ }^{3} J(\mathrm{H}, \mathrm{H})=7.4 \mathrm{~Hz}, \mathrm{Ar}-H\right), 8.24(\mathrm{~m}, 1 \mathrm{H}$, 
$\operatorname{Ar}-H), 8.39\left(\mathrm{~d}, 1 \mathrm{H},{ }^{3} \mathrm{~J}(\mathrm{H}, \mathrm{H})=7.4 \mathrm{~Hz}, \mathrm{Ar}-H\right) .{ }^{13} \mathrm{C}\left\{{ }^{1} \mathrm{H},{ }^{13} \mathrm{C}\right\} \mathrm{NMR}\left(\mathrm{CDCl}_{3}, 100 \mathrm{MHz}, 298 \mathrm{~K}\right)$ : $\delta(\mathrm{ppm})=31.1,31.6,32.4,32.5,32.6,32.7,35.2,35.4,38.2,38.5\left(\left(\mathrm{CH}_{3}\right)_{3} \mathrm{C}\right), 110.9,110.2$, $117.5,117.6,118.6,119.3,120.3,120.5,122.6,122.8,126.1,126.7,127.4,128.1,128.2$, 134.1, 134.2, 134.7, 134.8, 137.0, 138.1, 138.2, 138.5, 138.7, 139.0, 139.5, 139.6, 143.0, 143.5, 143.8, 151.1, 151.5, 154.2, 154.4, (Ar-C) 168.8, $169.8(\mathrm{P}=C) .{ }^{31} \mathrm{P}\left\{{ }^{1} \mathrm{H},{ }^{13} \mathrm{C}\right\} \mathrm{NMR}$ $\left(\mathrm{CDCl}_{3}, 162 \mathrm{MHz}, 298 \mathrm{~K}\right): \delta(\mathrm{ppm})=255.3,258.5$. NMR data for $\mathbf{1 b}:{ }^{1} \mathrm{H} \mathrm{NMR}\left(\mathrm{CDCl}_{3}, 400\right.$ $\mathrm{MHz}, 298 \mathrm{~K}): \delta(\mathrm{ppm})=1.41\left(\mathrm{~s}, 36 \mathrm{H},\left(\mathrm{CH}_{3}\right)_{3} \mathrm{C}\right), 1.52\left(\mathrm{~s}, 18 \mathrm{H},\left(\mathrm{CH}_{3}\right)_{3} \mathrm{C}\right), 5.9(\mathrm{~s}, 2 \mathrm{H}, \mathrm{Ar}-\mathrm{H})$, 7.02-7.04 (m, $2 \mathrm{H}, \mathrm{Ar}-H), 7.17-7.23(\mathrm{~m}, 4 \mathrm{H}, \mathrm{Ar}-H), 7.63(\mathrm{~s}, 4 \mathrm{H}, \mathrm{Ar}-H), 8.13\left(\mathrm{~d},{ }^{3} J(\mathrm{H}, \mathrm{H})=\right.$ $6.5 \mathrm{~Hz}, 2 \mathrm{H}) .{ }^{13} \mathrm{C}\left\{{ }^{1} \mathrm{H},{ }^{13} \mathrm{C}\right\} \mathrm{NMR}\left(\mathrm{CDCl}_{3}, 100 \mathrm{MHz}, 298 \mathrm{~K}\right): \delta(\mathrm{ppm})=31.6,32.6,35.4,38.4$ $\left(\mathrm{CH}_{3}\right)_{3} \mathrm{C}, 117.1,119.2,122.9,126.5,127.4,138.2,138.3,138.4,138.5,138.6,143.5,151.2$, 154.4 (Ar-C), $168.3(\mathrm{P}=C) .{ }^{31} \mathrm{P}\left\{{ }^{1} \mathrm{H},{ }^{13} \mathrm{C}\right\} \mathrm{NMR}\left(\mathrm{CDCl}_{3}, 162 \mathrm{MHz}, 298 \mathrm{~K}\right): \delta(\mathrm{ppm})=261.1$. Elemental analysis cacld for $\mathrm{C}_{56} \mathrm{H}_{68} \mathrm{P}_{2}(\%)$ : $\mathrm{C} 83.75 ; \mathrm{H} 8.53$; found: $\mathrm{C} 83.46 ; \mathrm{H}$ 8.92.

Synthesis of the radical anion $\left[(18-\mathrm{c}-6) \mathrm{K}(\mathrm{THF})_{2}\right]^{+} \cdot \mathbf{1}^{\bullet-}:$ Compound $\mathbf{1 a}$ or the mixture of $\mathbf{1 a}$ and 1 b (138 mg, $0.17 \mathrm{mmol}), \mathrm{K}(6.7 \mathrm{mg}, 0.17 \mathrm{mmol})$ and 18-crown-6 (53 mg, $0.20 \mathrm{mmol}$ ) were placed in a Schlenk flask, THF $(30 \mathrm{~mL})$ was transferred to the flask at room temperature via cannula with stirring. The mixture turned dark green after stirring for 6 hours. The solution was filtered and the filtrate was concentrated to ca. $10 \mathrm{~mL}$, and left at $-20{ }^{\circ} \mathrm{C}$ for 48 hours affording compound $\left[(18-\mathrm{c}-6) \mathrm{K}(\mathrm{THF})_{2}\right]^{+} \mathbf{1}^{*-}$ as dark-green crystals. Yield: $152 \mathrm{mg}, 71.6 \%$. Elemental analysis cacld for $\mathrm{C}_{76} \mathrm{H}_{108} \mathrm{KO}_{8} \mathrm{P}_{2}(\%)$ : $\mathrm{C} 72.98$; $\mathrm{H}$ 8.70; found: $\mathrm{C} 72.16 ; \mathrm{H} 8.31$.

Synthesis of the singlet diradical dianion $\left[(18-\mathrm{c}-6) \mathrm{K}(\mathrm{THF})_{2}\right]_{2}{ }^{2+} \cdot \mathbf{1}^{2-* *}$ : Compound 1a or the mixture of $1 \mathbf{a}$ and $\mathbf{1 b}$ (107 mg, $0.13 \mathrm{mmol}), \mathrm{KC}_{8}(54 \mathrm{mg}, 0.40 \mathrm{mmol})$ and 18-crown-6 (79 mg, $0.30 \mathrm{mmol})$ were placed in a Schlenk flask, THF $(40 \mathrm{~mL})$ was transferred to the flask at -78 ${ }^{\circ} \mathrm{C}$ via cannula with stirring. The mixture turned dark-green immediately after the addition of solvent. It was left to warm up to room temperature and stirred for 12 hours. The formed brown solution was filtered and the filtrate was concentrated to ca. $10 \mathrm{~mL}$, and left at $-20{ }^{\circ} \mathrm{C}$ for 48 hours affording compound $\left[(18-\mathrm{c}-6) \mathrm{K}(\mathrm{THF})_{2}\right]_{2}{ }^{2+} \cdot \mathbf{1}^{2--*}$ as dark-brown crystals. Yield: $122 \mathrm{mg}, 55.3 \%$. Elemental analysis cacld for $\mathrm{C}_{96} \mathrm{H}_{148} \mathrm{~K}_{2} \mathrm{O}_{16} \mathrm{P}_{2}(\%)$ : C 67.89; H 8.78; found: $\mathrm{C}$ $68.25 ; \mathrm{H} 8.38$. 
(a)

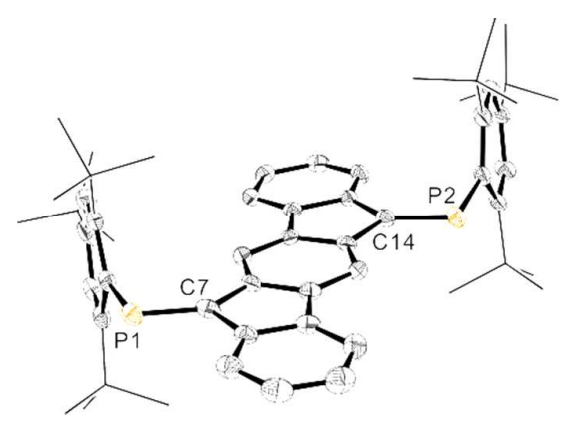

(b)

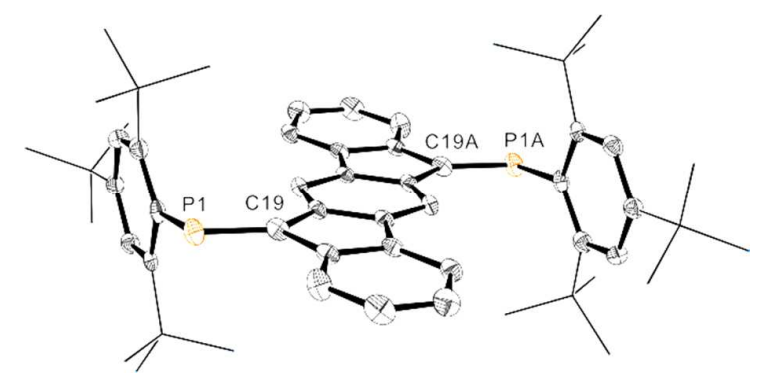

Figure S1. The solid state structures of $1 \mathrm{a}(\mathrm{a})$ and $\mathbf{1 b}(\mathrm{b})$.

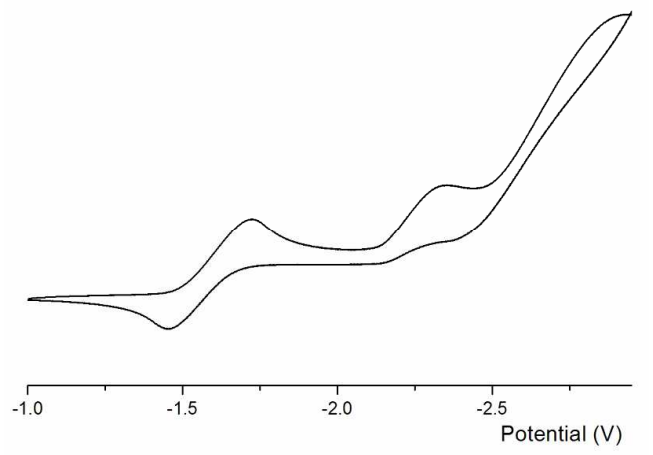

Figure S2. The cyclic voltammogram of $1 \mathbf{a}\left(1 \times 10^{-3} \mathrm{M}\right)$ in THF solution at room temperature, containing $0.05 \mathrm{M} n \mathrm{Bu}_{4} \mathrm{PF}_{6}$, measured at $100 \mathrm{mV} / \mathrm{s}$.

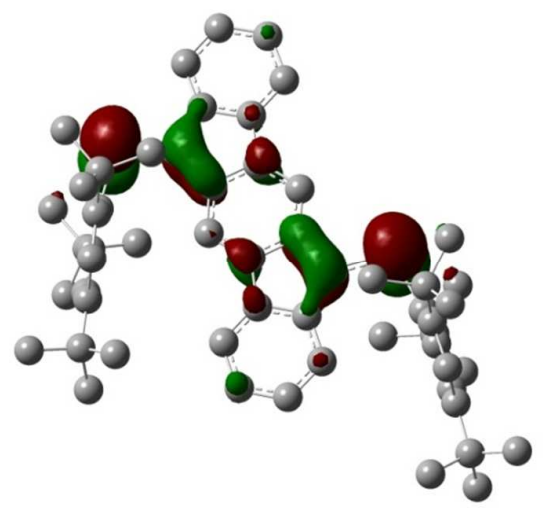

Figure S3. LUMO of 1a calculated at the WB97XD/6-31G(d) level of theory. 


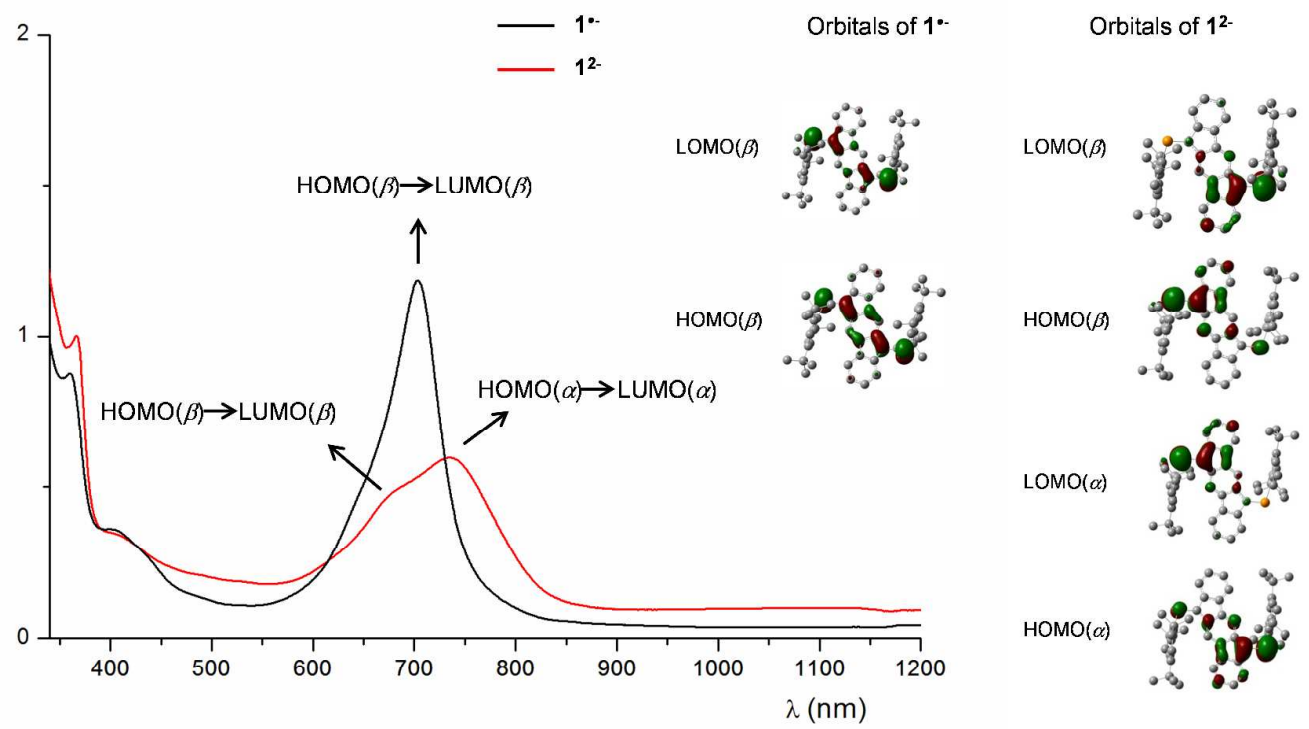

Figure S4. UV-vis absorption spectra of $\left[(18-\mathrm{c}-6) \mathrm{K}(\mathrm{THF})_{2}\right] \cdot \mathbf{1}^{--}$(black curve) and $\left[(18-\mathrm{c}-6) \mathrm{K}(\mathrm{THF})_{2}\right]_{2} \cdot \mathbf{1}^{2-*}$ (red curve) in THF at room temperature.

(a)

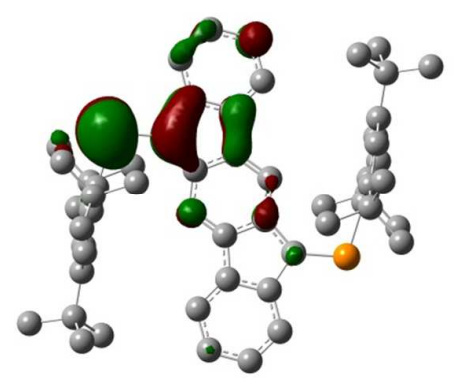

(b)

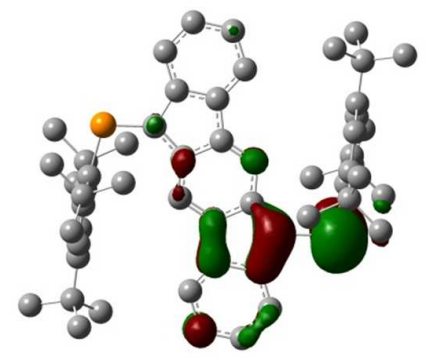

Figure S5. The $\operatorname{SOMO}(\alpha)\left(\right.$ a) and $\operatorname{SOMO}(\beta)$ (b) of $\mathbf{1}^{2-\cdots}$ calculated at the $\operatorname{UWB} 97 \mathrm{XD} / 6-31 \mathrm{G}(\mathrm{d})$ level of theory. 
Table S1. Crystal Data and Structure Refinement

\begin{tabular}{|c|c|c|c|c|}
\hline & $1 \mathrm{a}$ & $1 b$ & $\begin{array}{l}{\left[(18-\mathrm{c}-6) \mathrm{K}(\mathrm{THF})_{2}\right.} \\
] \cdot \mathbf{1}^{\bullet-}\end{array}$ & $\begin{array}{l}{\left[(18-\mathrm{c}-6) \mathrm{K}(\mathrm{THF})_{2}\right.} \\
]_{2} \cdot \mathbf{1}^{2-\bullet}\end{array}$ \\
\hline formula & $\mathrm{C}_{119} \mathrm{H}_{152} \mathrm{P}_{4}$ & $\mathrm{C}_{56} \mathrm{H}_{68} \mathrm{P}_{2}$ & $\mathrm{C}_{76} \mathrm{H}_{108} \mathrm{KO}_{8} \mathrm{P}_{2}$ & $\mathrm{C}_{112} \mathrm{H}_{180} \mathrm{~K}_{2} \mathrm{O}_{20} \mathrm{P}_{2}$ \\
\hline formula weight & 1706.29 & 803.04 & 1250.66 & 1986.70 \\
\hline crystal system & Monoclinic & Monoclinic & Monoclinic & triclinic \\
\hline space group & $P 2_{1} / n$ & $C 2 / c$ & $P 2_{1} / n$ & $P-1$ \\
\hline$a / \AA$ & $13.840(3)$ & $30.270(6)$ & $13.392(3)$ & $13.4139(7)$ \\
\hline$b / \AA$ & $26.967(5)$ & $9.0060(18)$ & $17.887(4)$ & $13.5562(7)$ \\
\hline $\mathrm{c} / \AA$ & $14.534(3)$ & $17.970(4)$ & $15.880(3)$ & $17.2567(9)$ \\
\hline$\alpha / \operatorname{deg}$ & & & & $102.9195(15)$ \\
\hline$\beta /$ deg & $95.30(3)$ & $102.24(3)$ & $107.74(3)$ & $104.8459(14)$ \\
\hline$\gamma / \operatorname{deg}$ & & & & $96.8203(14)$ \\
\hline$V / \AA^{3}$ & $5401.3(19)$ & $4787.5(17)$ & $3623.2(13)$ & $2904.4(3)$ \\
\hline$Z$ & 2 & 4 & 2 & 1 \\
\hline$\rho_{\text {calcd }} / \mathrm{g} \cdot \mathrm{cm}^{-3}$ & 1.049 & 1.114 & 1.146 & 1.136 \\
\hline crystal size $/ \mathrm{mm}^{3}$ & $0.28 \times 0.22 \times 0.19$ & $0.12 \times 0.10 \times 0.08$ & $0.06 \times 0.04 \times 0.04$ & $0.18 \times 0.08 \times 0.06$ \\
\hline$\theta$ range/deg & $2.09-25.00$ & $2.32-24.99$ & $2.09-25.00$ & $2.02-25.00$ \\
\hline \multirow[t]{3}{*}{ index ranges } & $-12 \leq \mathrm{h} \leq 16$ & $-35 \leq h \leq 35$ & $-15 \leq h \leq 15$ & $-15 \leq h \leq 15$ \\
\hline & $-32 \leq k \leq 26$ & $-7 \leq k \leq 10$ & $-19 \leq k \leq 21$ & $-15 \leq k \leq 16$ \\
\hline & $-17 \leq l \leq 16$ & $-21 \leq l \leq 21$ & $-18 \leq l \leq 17$ & $-20 \leq l \leq 14$ \\
\hline collected data & 18626 & 16498 & 20224 & 20386 \\
\hline unique data & $\begin{array}{l}9500\left(R_{\text {int }}=\right. \\
0.0859)\end{array}$ & $\begin{array}{l}4217\left(R_{\text {int }}=\right. \\
0.0707)\end{array}$ & $\begin{array}{l}6322\left(\mathrm{R}_{\text {int }}=\right. \\
0.1765)\end{array}$ & $\begin{array}{l}10119\left(\mathrm{R}_{\text {int }}=\right. \\
0.0285)\end{array}$ \\
\hline completeness to $\theta$ & $99.7 \%$ & $99.9 \%$ & $99.1 \%$ & $99.1 \%$ \\
\hline data/restraints/parameters & $9500 / 166$ / 603 & 4217 / 0 / 271 & $6322 / 93 / 421$ & 10119 / 13 / 625 \\
\hline GOF on $F^{2}$ & 1.041 & 1.055 & 1.013 & 1.092 \\
\hline \multirow[t]{2}{*}{ final $R$ indices $[I>2 \square(I)]$} & $R_{1}=0.0937$ & $R_{1}=0.0582$ & $R_{1}=0.0883$ & $R_{1}=0.0576$ \\
\hline & $w R_{2}=0.2474$ & $w R_{2}=0.1262$ & $w R_{2}=0.1865$ & $w R_{2}=0.1495$ \\
\hline $\begin{array}{l}\text { Largest diff peak/hole } \\
\left(\mathrm{e} \cdot \AA^{-3}\right)\end{array}$ & $1.173 /-0.432$ & $0.453 /-0.282$ & $0.511 /-0.680$ & $0.901 /-0.684$ \\
\hline
\end{tabular}


Table S2. Selected experimental and calculated bond lengths $(\AA)$ and relative energy $(\mathrm{kcal} / \mathrm{mol})$ of $\mathbf{1}^{2-*}$.

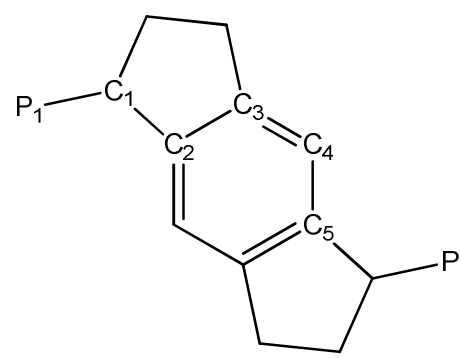

\begin{tabular}{|l|l|l|l|l|l|l|}
\hline & Energy $(\mathrm{kcal} / \mathrm{mol})^{b}$ & $\mathrm{P} 1-\mathrm{C} 1$ & $\mathrm{C} 1-\mathrm{C} 2$ & $\mathrm{C} 2-\mathrm{C} 3$ & $\mathrm{C} 3-\mathrm{C} 4$ & C4-C5 \\
\hline X-ray & & $1.768(2)$ & $1.429(3)$ & $1.457(3)$ & $1.383(3)$ & $1.409(3)$ \\
\hline $\mathrm{CS}^{a}$ & 10.2 & 1.775 & 1.409 & 1.457 & 1.365 & 1.426 \\
\hline $\mathrm{OS}^{a}$ & 0 & 1.764 & 1.446 & 1.437 & 1.384 & 1.406 \\
\hline $\mathrm{T}^{a}$ & 6.7 & 1.762 & 1.462 & 1.430 & 1.391 & 1.399 \\
\hline
\end{tabular}

${ }^{a} \mathrm{CS}$ : close-shell singlet; OS: open-shell singlet; T: triplet.

${ }^{b}$ The approximate spin projection method is applied. 


\section{Calculation details}

All the geometry optimizations were carried out at the (U)WB97XD/6-31G(d) level of theory. The obtained stationary points were characterized by frequency calculations. The molecular orbitals and spin densities were calculated at the level of (U)WB97XD/6-31G(d) at the optimized geometries. The UV-vis absorption spectra were calculated using time-dependent DFT (TD-DFT) method and polarized continuum model (PCM) was adopted to consider solvent (THF) effects. All calculations were performed with the Gaussian 09 program suite. ${ }^{3}$

Table S3. Cartesian coordinates $(\mathrm{x}, \mathrm{y}, \mathrm{z})$ for the optimized structure of 1a.

\begin{tabular}{|c|c|c|c|}
\hline $\mathrm{P}$ & -5.33207400 & 1.24185400 & -0.16682900 \\
\hline $\mathrm{P}$ & 2.96605500 & 2.11390700 & 0.24770700 \\
\hline $\mathrm{C}$ & -3.81850500 & 3.53717000 & -0.03827000 \\
\hline $\mathrm{C}$ & -4.84529300 & 4.47563400 & -0.05288900 \\
\hline $\mathrm{H}$ & -5.88164600 & 4.16572800 & -0.15456400 \\
\hline $\mathrm{C}$ & -4.52575400 & 5.82674300 & 0.06406400 \\
\hline $\mathrm{H}$ & -5.31891400 & 6.56814100 & 0.05148800 \\
\hline $\mathrm{C}$ & -3.19860400 & 6.23773600 & 0.19899300 \\
\hline $\mathrm{H}$ & -2.97033600 & 7.29516300 & 0.29378000 \\
\hline $\mathrm{C}$ & -2.16573200 & 5.30280300 & 0.21502600 \\
\hline $\mathrm{H}$ & -1.13339400 & 5.62467400 & 0.32114600 \\
\hline $\mathrm{C}$ & -2.48179400 & 3.95601400 & 0.09345300 \\
\hline $\mathrm{C}$ & -3.86006600 & 2.05353800 & -0.11833900 \\
\hline $\mathrm{C}$ & -2.44849300 & 1.62545200 & -0.07765700 \\
\hline $\mathrm{C}$ & -1.62263800 & 2.76773800 & 0.06282900 \\
\hline $\mathrm{C}$ & -0.23905300 & 2.67105800 & 0.12508500 \\
\hline $\mathrm{H}$ & 0.37734500 & 3.55913900 & 0.23655700 \\
\hline $\mathrm{C}$ & 0.32962300 & 1.40290200 & 0.02042500 \\
\hline $\mathrm{C}$ & -0.49116900 & 0.27350400 & -0.15000200 \\
\hline $\mathrm{C}$ & -1.87455400 & 0.36136800 & -0.18685500 \\
\hline $\mathrm{H}$ & -2.47278300 & -0.53062600 & -0.31121300 \\
\hline $\mathrm{C}$ & 1.75153800 & 0.97225600 & 0.02919800 \\
\hline $\mathrm{C}$ & 1.71657200 & -0.49606500 & -0.17341800 \\
\hline $\mathrm{C}$ & 0.36906000 & -0.90090100 & -0.28204700 \\
\hline $\mathrm{C}$ & 0.02260100 & -2.22577600 & -0.49920200 \\
\hline $\mathrm{H}$ & -1.02249600 & -2.50706500 & -0.59807100 \\
\hline $\mathrm{C}$ & 1.03668700 & -3.17593700 & -0.59254000 \\
\hline $\mathrm{H}$ & 0.78561500 & -4.22015000 & -0.75777100 \\
\hline $\mathrm{C}$ & 2.37215200 & -2.79051200 & -0.47508500 \\
\hline $\mathrm{H}$ & 3.15625600 & -3.53847100 & -0.54740700 \\
\hline $\mathrm{C}$ & 2.72387000 & -1.45636500 & -0.26943900 \\
\hline $\mathrm{H}$ & 3.76990700 & -1.19323300 & -0.18896100 \\
\hline $\mathrm{C}$ & -4.89084400 & -0.55823500 & -0.07829500 \\
\hline
\end{tabular}




\begin{tabular}{|c|c|c|c|}
\hline $\mathrm{C}$ & -4.68116200 & -1.31472700 & -1.26341000 \\
\hline $\mathrm{C}$ & -4.14033600 & -2.59966300 & -1.13947700 \\
\hline $\mathrm{H}$ & -3.94110200 & -3.16586100 & -2.03592200 \\
\hline $\mathrm{C}$ & -3.84394700 & -3.18484600 & 0.08365800 \\
\hline $\mathrm{C}$ & -4.16863400 & -2.46031300 & 1.22474100 \\
\hline $\mathrm{H}$ & -4.00066300 & -2.93280200 & 2.18057000 \\
\hline $\mathrm{C}$ & -4.70127600 & -1.17178700 & 1.19559300 \\
\hline $\mathrm{C}$ & -4.98102800 & -0.81455700 & -2.70404200 \\
\hline $\mathrm{C}$ & -6.39448900 & -0.20752000 & -2.80718500 \\
\hline $\mathrm{H}$ & -6.51727200 & 0.71230100 & -2.23060500 \\
\hline $\mathrm{H}$ & -7.14831500 & -0.92396000 & -2.46277600 \\
\hline $\mathrm{H}$ & -6.61160100 & 0.04239800 & -3.85168800 \\
\hline $\mathrm{C}$ & -4.95931700 & -1.97099800 & -3.72680300 \\
\hline $\mathrm{H}$ & -5.64063900 & -2.78153100 & -3.44570500 \\
\hline $\mathrm{H}$ & -3.95748800 & -2.38809900 & -3.87202600 \\
\hline $\mathrm{H}$ & -5.28430900 & -1.58500100 & -4.69835900 \\
\hline $\mathrm{C}$ & -3.91502300 & 0.19690600 & -3.17177200 \\
\hline $\mathrm{H}$ & -4.08143500 & 0.44805300 & -4.22591000 \\
\hline $\mathrm{H}$ & -2.91191200 & -0.23332200 & -3.07848600 \\
\hline $\mathrm{H}$ & -3.93571700 & 1.12609500 & -2.60099200 \\
\hline $\mathrm{C}$ & -3.29620300 & -4.61328900 & 0.20584800 \\
\hline $\mathrm{C}$ & -2.69496500 & -5.11927400 & -1.11510100 \\
\hline $\mathrm{H}$ & -3.45113000 & -5.21753400 & -1.90111700 \\
\hline $\mathrm{H}$ & -2.25620400 & -6.11134400 & -0.96327900 \\
\hline $\mathrm{H}$ & -1.90434900 & -4.45580600 & -1.48411600 \\
\hline $\mathrm{C}$ & -4.46591400 & -5.53818800 & 0.59750400 \\
\hline $\mathrm{H}$ & -5.25303000 & -5.51400300 & -0.16383200 \\
\hline $\mathrm{H}$ & -4.90956700 & -5.22967500 & 1.55024700 \\
\hline $\mathrm{H}$ & -4.11898400 & -6.57321400 & 0.70207000 \\
\hline $\mathrm{C}$ & -2.20675200 & -4.69931000 & 1.29176600 \\
\hline $\mathrm{H}$ & -1.38185400 & -4.00825400 & 1.09250600 \\
\hline $\mathrm{H}$ & -1.79851900 & -5.71569800 & 1.32769900 \\
\hline $\mathrm{H}$ & -2.59809600 & -4.47530300 & 2.28883600 \\
\hline $\mathrm{C}$ & -5.04026900 & -0.52398500 & 2.57032000 \\
\hline $\mathrm{C}$ & -6.46058600 & 0.07563000 & 2.57698800 \\
\hline $\mathrm{H}$ & -6.57967000 & 0.92001900 & 1.89397900 \\
\hline $\mathrm{H}$ & -6.69896100 & 0.44424800 & 3.58093100 \\
\hline $\mathrm{H}$ & -7.20223200 & -0.68351400 & 2.30521600 \\
\hline $\mathrm{C}$ & -5.03883000 & -1.57022500 & 3.70674300 \\
\hline $\mathrm{H}$ & -5.70559200 & -2.41300300 & 3.49342100 \\
\hline $\mathrm{H}$ & -5.39432400 & -1.09020400 & 4.62394100 \\
\hline $\mathrm{H}$ & -4.03758200 & -1.95833700 & 3.92056900 \\
\hline $\mathrm{C}$ & -3.99448800 & 0.53884100 & 2.96840800 \\
\hline $\mathrm{H}$ & -2.98160400 & 0.13111000 & 2.88267600 \\
\hline
\end{tabular}




\begin{tabular}{|c|c|c|c|}
\hline $\mathrm{H}$ & -4.15552900 & 0.83926200 & 4.01038600 \\
\hline $\mathrm{H}$ & -4.04820800 & 1.43732400 & 2.35377000 \\
\hline $\mathrm{C}$ & 4.53533200 & 1.13863000 & 0.15575200 \\
\hline $\mathrm{C}$ & 5.14953000 & 0.90394100 & -1.10336800 \\
\hline $\mathrm{C}$ & 6.16173000 & -0.05971400 & -1.16945700 \\
\hline $\mathrm{H}$ & 6.60779800 & -0.27898500 & -2.12673000 \\
\hline $\mathrm{C}$ & 6.61333300 & -0.76039500 & -0.06168600 \\
\hline $\mathrm{C}$ & 6.08381000 & -0.40540300 & 1.17499100 \\
\hline $\mathrm{H}$ & 6.47753100 & -0.90276100 & 2.05044600 \\
\hline $\mathrm{C}$ & 5.07362400 & 0.54152000 & 1.33237200 \\
\hline $\mathrm{C}$ & 4.77795000 & 1.62516000 & -2.43037400 \\
\hline $\mathrm{C}$ & 4.67898000 & 3.15108000 & -2.23390400 \\
\hline $\mathrm{H}$ & 4.50873900 & 3.63617700 & -3.20145100 \\
\hline $\mathrm{H}$ & 5.60845900 & 3.54776100 & -1.81080800 \\
\hline $\mathrm{H}$ & 3.85742500 & 3.45447800 & -1.58047200 \\
\hline $\mathrm{C}$ & 5.86623200 & 1.42457100 & -3.50788700 \\
\hline $\mathrm{H}$ & 5.91579400 & 0.39296800 & -3.87106300 \\
\hline $\mathrm{H}$ & 6.85888800 & 1.71934600 & -3.15032300 \\
\hline $\mathrm{H}$ & 5.62334500 & 2.05082900 & -4.37242500 \\
\hline $\mathrm{C}$ & 3.46992100 & 1.06508000 & -3.02682400 \\
\hline $\mathrm{H}$ & 3.52695400 & -0.02469700 & -3.12124900 \\
\hline $\mathrm{H}$ & 3.31152400 & 1.48726300 & -4.02630700 \\
\hline $\mathrm{H}$ & 2.59307800 & 1.30391100 & -2.42409400 \\
\hline $\mathrm{C}$ & 7.67296500 & -1.86418800 & -0.14860100 \\
\hline $\mathrm{C}$ & 8.12671500 & -2.12687600 & -1.59107600 \\
\hline $\mathrm{H}$ & 7.29177100 & -2.43648300 & -2.22949300 \\
\hline $\mathrm{H}$ & 8.86913200 & -2.93204400 & -1.60119000 \\
\hline $\mathrm{H}$ & 8.59405600 & -1.24264300 & -2.03823900 \\
\hline $\mathrm{C}$ & 8.90756700 & -1.45935500 & 0.67909200 \\
\hline $\mathrm{H}$ & 9.67265400 & -2.24281000 & 0.62865400 \\
\hline $\mathrm{H}$ & 8.65914800 & -1.30359300 & 1.73383800 \\
\hline $\mathrm{H}$ & 9.34337400 & -0.53032300 & 0.29586000 \\
\hline $\mathrm{C}$ & 7.08140900 & -3.17106500 & 0.41411600 \\
\hline $\mathrm{H}$ & 6.19795700 & -3.47490000 & -0.15873900 \\
\hline $\mathrm{H}$ & 6.78143900 & -3.06335700 & 1.46131300 \\
\hline $\mathrm{H}$ & 7.82090900 & -3.97836800 & 0.35886400 \\
\hline $\mathrm{C}$ & 4.61298700 & 0.85531700 & 2.78334200 \\
\hline $\mathrm{C}$ & 4.53439200 & 2.37519200 & 3.02877100 \\
\hline $\mathrm{H}$ & 5.49548200 & 2.85244300 & 2.80820300 \\
\hline $\mathrm{H}$ & 4.29117800 & 2.56589900 & 4.08001900 \\
\hline $\mathrm{H}$ & 3.76682500 & 2.87364300 & 2.43169000 \\
\hline $\mathrm{C}$ & 3.26406300 & 0.17897200 & 3.10405600 \\
\hline $\mathrm{H}$ & 3.03927300 & 0.29590500 & 4.17077200 \\
\hline $\mathrm{H}$ & 3.30881100 & -0.89213100 & 2.87943900 \\
\hline
\end{tabular}




$\begin{array}{lrrr}\mathrm{H} & 2.43254500 & 0.60516300 & 2.54198500 \\ \mathrm{C} & 5.62171700 & 0.32441800 & 3.82466000 \\ \mathrm{H} & 6.63810700 & 0.68721800 & 3.63643100 \\ \mathrm{H} & 5.64165900 & -0.76933700 & 3.87171500 \\ \mathrm{H} & 5.32091000 & 0.67715400 & 4.81645000\end{array}$

Table S4. Cartesian coordinates $(\mathrm{x}, \mathrm{y}, \mathrm{z})$ for the optimized structure of $1^{\circ}$.

\begin{tabular}{|c|c|c|c|}
\hline $\mathrm{P}$ & -3.53205000 & -1.54957200 & 1.96119100 \\
\hline $\mathrm{C}$ & -2.32464900 & -1.45748000 & 0.72331300 \\
\hline $\mathrm{C}$ & -1.18053500 & -0.62675900 & 0.48715100 \\
\hline $\mathrm{C}$ & -0.54317900 & -1.01863700 & -0.73996600 \\
\hline $\mathrm{C}$ & -1.31902400 & -2.10907400 & -1.30686900 \\
\hline $\mathrm{C}$ & -2.38325200 & -2.39125800 & -0.42288000 \\
\hline $\mathrm{C}$ & -3.28391100 & -3.40969800 & -0.74066300 \\
\hline $\mathrm{H}$ & -4.11117300 & -3.64250300 & -0.07501300 \\
\hline $\mathrm{C}$ & -3.11468400 & -4.12394300 & -1.92448200 \\
\hline $\mathrm{H}$ & -3.81475000 & -4.91712700 & -2.17511700 \\
\hline $\mathrm{C}$ & -2.06408700 & -3.82794100 & -2.80094800 \\
\hline $\mathrm{H}$ & -1.95385000 & -4.38846600 & -3.72644800 \\
\hline $\mathrm{C}$ & -1.16232500 & -2.81333800 & -2.49496200 \\
\hline $\mathrm{H}$ & -0.34908700 & -2.55953700 & -3.17074900 \\
\hline $\mathrm{C}$ & -0.60117500 & 0.42962300 & 1.22182700 \\
\hline $\mathrm{H}$ & -1.03743800 & 0.78180600 & 2.14753300 \\
\hline $\mathrm{C}$ & -3.15802400 & -0.08029800 & 3.05831100 \\
\hline $\mathrm{C}$ & -2.27590100 & -0.19420500 & 4.17067900 \\
\hline $\mathrm{C}$ & -1.86628900 & 0.97264300 & 4.82733400 \\
\hline $\mathrm{H}$ & -1.15599400 & 0.89280200 & 5.63520800 \\
\hline $\mathrm{C}$ & -2.31677600 & 2.23755000 & 4.47969900 \\
\hline $\mathrm{C}$ & -3.26906800 & 2.30875800 & 3.47078700 \\
\hline $\mathrm{H}$ & -3.67046800 & 3.28287500 & 3.23449800 \\
\hline $\mathrm{C}$ & -3.72048800 & 1.19796500 & 2.75655800 \\
\hline $\mathrm{C}$ & -4.81779900 & 1.46314500 & 1.68285400 \\
\hline $\mathrm{C}$ & -6.04733400 & 0.56381700 & 1.92288100 \\
\hline $\mathrm{H}$ & -6.46238900 & 0.74055100 & 2.92246500 \\
\hline $\mathrm{H}$ & -5.81051100 & -0.49776500 & 1.83158400 \\
\hline $\mathrm{H}$ & -6.82447400 & 0.79655200 & 1.18408700 \\
\hline $\mathrm{C}$ & -4.27337300 & 1.28413200 & 0.25016600 \\
\hline $\mathrm{H}$ & -4.07682200 & 0.24165100 & 0.00571100 \\
\hline $\mathrm{H}$ & -3.33755900 & 1.83839900 & 0.12235700 \\
\hline $\mathrm{H}$ & -5.00465900 & 1.66960700 & -0.47179100 \\
\hline $\mathrm{C}$ & -5.35368000 & 2.91041200 & 1.74844500 \\
\hline $\mathrm{H}$ & -6.17630000 & 3.00720900 & 1.03147300 \\
\hline $\mathrm{H}$ & -4.59641500 & 3.65060300 & 1.46783700 \\
\hline $\mathrm{H}$ & -5.74765200 & 3.16553100 & 2.73904800 \\
\hline
\end{tabular}




\begin{tabular}{|c|c|c|c|}
\hline $\mathrm{C}$ & -1.88963000 & 3.50624200 & 5.23173500 \\
\hline $\mathrm{C}$ & -3.02873200 & 3.90310100 & 6.19116300 \\
\hline $\mathrm{H}$ & -3.95757600 & 4.09033300 & 5.64127100 \\
\hline $\mathrm{H}$ & -2.76916300 & 4.81460700 & 6.74503400 \\
\hline $\mathrm{H}$ & -3.22332200 & 3.10348800 & 6.91485200 \\
\hline $\mathrm{C}$ & -0.61023500 & 3.29081800 & 6.05572300 \\
\hline $\mathrm{H}$ & -0.75672900 & 2.56216000 & 6.86042900 \\
\hline $\mathrm{H}$ & -0.31027100 & 4.23571400 & 6.52346000 \\
\hline $\mathrm{H}$ & 0.22035800 & 2.94579000 & 5.43018700 \\
\hline $\mathrm{C}$ & -1.63222300 & 4.66970100 & 4.25496700 \\
\hline $\mathrm{H}$ & -0.86340300 & 4.41405800 & 3.51992300 \\
\hline $\mathrm{H}$ & -1.29435200 & 5.55352400 & 4.80983400 \\
\hline $\mathrm{H}$ & -2.53596300 & 4.95675900 & 3.70837300 \\
\hline $\mathrm{C}$ & -1.69983300 & -1.53423000 & 4.71260300 \\
\hline $\mathrm{C}$ & -2.83101900 & -2.54123900 & 5.00215500 \\
\hline $\mathrm{H}$ & -3.54095700 & -2.12269000 & 5.72580200 \\
\hline $\mathrm{H}$ & -2.40837100 & -3.45903900 & 5.42961200 \\
\hline $\mathrm{H}$ & -3.38224100 & -2.81804300 & 4.10161800 \\
\hline $\mathrm{C}$ & -0.66447900 & -2.13157900 & 3.73916100 \\
\hline $\mathrm{H}$ & -0.19391400 & -3.01228500 & 4.19480200 \\
\hline $\mathrm{H}$ & 0.11910700 & -1.39911600 & 3.51714300 \\
\hline $\mathrm{H}$ & -1.11052100 & -2.43717400 & 2.79323800 \\
\hline $\mathrm{C}$ & -0.96363000 & -1.34896000 & 6.05713400 \\
\hline $\mathrm{H}$ & -1.59913500 & -0.88152400 & 6.81826400 \\
\hline $\mathrm{H}$ & -0.04497500 & -0.76113000 & 5.95532200 \\
\hline $\mathrm{H}$ & -0.66978000 & -2.33564200 & 6.43198900 \\
\hline $\mathrm{P}$ & 3.53205000 & 1.54957200 & -1.96119100 \\
\hline $\mathrm{C}$ & 2.32464900 & 1.45748000 & -0.72331300 \\
\hline $\mathrm{C}$ & 1.18053500 & 0.62675900 & -0.48715100 \\
\hline $\mathrm{C}$ & 0.54317900 & 1.01863700 & 0.73996600 \\
\hline $\mathrm{C}$ & 1.31902400 & 2.10907400 & 1.30686900 \\
\hline $\mathrm{C}$ & 2.38325200 & 2.39125800 & 0.42288000 \\
\hline $\mathrm{C}$ & 3.28391100 & 3.40969800 & 0.74066300 \\
\hline $\mathrm{H}$ & 4.11117300 & 3.64250300 & 0.07501300 \\
\hline $\mathrm{C}$ & 3.11468400 & 4.12394300 & 1.92448200 \\
\hline $\mathrm{H}$ & 3.81475000 & 4.91712700 & 2.17511700 \\
\hline $\mathrm{C}$ & 2.06408700 & 3.82794100 & 2.80094800 \\
\hline $\mathrm{H}$ & 1.95385000 & 4.38846600 & 3.72644800 \\
\hline $\mathrm{C}$ & 1.16232500 & 2.81333800 & 2.49496200 \\
\hline $\mathrm{H}$ & 0.34908700 & 2.55953700 & 3.17074900 \\
\hline $\mathrm{C}$ & 0.60117500 & -0.42962300 & -1.22182700 \\
\hline $\mathrm{H}$ & 1.03743800 & -0.78180600 & -2.14753300 \\
\hline $\mathrm{C}$ & 3.15802400 & 0.08029800 & -3.05831100 \\
\hline $\mathrm{C}$ & 2.27590100 & 0.19420500 & -4.17067900 \\
\hline
\end{tabular}




\begin{tabular}{|c|c|c|c|}
\hline $\mathrm{C}$ & 1.86628900 & -0.97264300 & -4.82733400 \\
\hline $\mathrm{H}$ & 1.15599400 & -0.89280200 & -5.63520800 \\
\hline $\mathrm{C}$ & 2.31677600 & -2.23755000 & -4.47969900 \\
\hline $\mathrm{C}$ & 3.26906800 & -2.30875800 & -3.47078700 \\
\hline $\mathrm{H}$ & 3.67046800 & -3.28287500 & -3.23449800 \\
\hline $\mathrm{C}$ & 3.72048800 & -1.19796500 & -2.75655800 \\
\hline $\mathrm{C}$ & 4.81779900 & -1.46314500 & -1.68285400 \\
\hline $\mathrm{C}$ & 6.04733400 & -0.56381700 & -1.92288100 \\
\hline $\mathrm{H}$ & 6.46238900 & -0.74055100 & -2.92246500 \\
\hline $\mathrm{H}$ & 5.81051100 & 0.49776500 & -1.83158400 \\
\hline $\mathrm{H}$ & 6.82447400 & -0.79655200 & -1.18408700 \\
\hline $\mathrm{C}$ & 4.27337300 & -1.28413200 & -0.25016600 \\
\hline $\mathrm{H}$ & 4.07682200 & -0.24165100 & -0.00571100 \\
\hline $\mathrm{H}$ & 3.33755900 & -1.83839900 & -0.12235700 \\
\hline $\mathrm{H}$ & 5.00465900 & -1.66960700 & 0.47179100 \\
\hline $\mathrm{C}$ & 5.35368000 & -2.91041200 & -1.74844500 \\
\hline $\mathrm{H}$ & 6.17630000 & -3.00720900 & -1.03147300 \\
\hline $\mathrm{H}$ & 4.59641500 & -3.65060300 & -1.46783700 \\
\hline $\mathrm{H}$ & 5.74765200 & -3.16553100 & -2.73904800 \\
\hline $\mathrm{C}$ & 1.88963000 & -3.50624200 & -5.23173500 \\
\hline $\mathrm{C}$ & 3.02873200 & -3.90310100 & -6.19116300 \\
\hline $\mathrm{H}$ & 3.95757600 & -4.09033300 & -5.64127100 \\
\hline $\mathrm{H}$ & 2.76916300 & -4.81460700 & -6.74503400 \\
\hline $\mathrm{H}$ & 3.22332200 & -3.10348800 & -6.91485200 \\
\hline $\mathrm{C}$ & 0.61023500 & -3.29081800 & -6.05572300 \\
\hline $\mathrm{H}$ & 0.75672900 & -2.56216000 & -6.86042900 \\
\hline $\mathrm{H}$ & 0.31027100 & -4.23571400 & -6.52346000 \\
\hline $\mathrm{H}$ & -0.22035800 & -2.94579000 & -5.43018700 \\
\hline $\mathrm{C}$ & 1.63222300 & -4.66970100 & -4.25496700 \\
\hline $\mathrm{H}$ & 0.86340300 & -4.41405800 & -3.51992300 \\
\hline $\mathrm{H}$ & 1.29435200 & -5.55352400 & -4.80983400 \\
\hline $\mathrm{H}$ & 2.53596300 & -4.95675900 & -3.70837300 \\
\hline $\mathrm{C}$ & 1.69983300 & 1.53423000 & -4.71260300 \\
\hline $\mathrm{C}$ & 2.83101900 & 2.54123900 & -5.00215500 \\
\hline $\mathrm{H}$ & 3.54095700 & 2.12269000 & -5.72580200 \\
\hline $\mathrm{H}$ & 2.40837100 & 3.45903900 & -5.42961200 \\
\hline $\mathrm{H}$ & 3.38224100 & 2.81804300 & -4.10161800 \\
\hline $\mathrm{C}$ & 0.66447900 & 2.13157900 & -3.73916100 \\
\hline $\mathrm{H}$ & 0.19391400 & 3.01228500 & -4.19480200 \\
\hline $\mathrm{H}$ & -0.11910700 & 1.39911600 & -3.51714300 \\
\hline $\mathrm{H}$ & 1.11052100 & 2.43717400 & -2.79323800 \\
\hline $\mathrm{C}$ & 0.96363000 & 1.34896000 & -6.05713400 \\
\hline $\mathrm{H}$ & 1.59913500 & 0.88152400 & -6.81826400 \\
\hline $\mathrm{H}$ & 0.04497500 & 0.76113000 & -5.95532200 \\
\hline
\end{tabular}


Table S5. Cartesian coordinates $(\mathrm{x}, \mathrm{y}, \mathrm{z})$ for the optimized structure of $\mathbf{1}^{2-*}$ featuring a close-shell singlet state.

\begin{tabular}{|c|c|c|c|}
\hline $\mathrm{P}$ & -3.00039400 & -3.14764100 & -0.44439700 \\
\hline $\mathrm{C}$ & -1.37738200 & -2.46084200 & -0.23328000 \\
\hline $\mathrm{C}$ & -0.83587700 & -1.16590300 & -0.11516000 \\
\hline $\mathrm{C}$ & -1.42041700 & 0.13437300 & -0.13189400 \\
\hline $\mathrm{H}$ & -2.49186100 & 0.26518700 & -0.22397800 \\
\hline $\mathrm{C}$ & -0.61267300 & 1.23013100 & -0.03233400 \\
\hline $\mathrm{C}$ & -0.97072300 & 2.63435600 & -0.02467700 \\
\hline $\mathrm{C}$ & -2.19978900 & 3.26872900 & -0.16914100 \\
\hline $\mathrm{H}$ & -3.09688000 & 2.67340000 & -0.32797300 \\
\hline $\mathrm{C}$ & -2.25687000 & 4.65956200 & -0.11735000 \\
\hline $\mathrm{H}$ & -3.21418100 & 5.16711300 & -0.22459100 \\
\hline $\mathrm{C}$ & -1.08373300 & 5.40406000 & 0.06546800 \\
\hline $\mathrm{H}$ & -1.13868500 & 6.49087100 & 0.10229200 \\
\hline $\mathrm{C}$ & 0.15265000 & 4.77360200 & 0.19208000 \\
\hline $\mathrm{H}$ & 1.06061200 & 5.35870600 & 0.31813100 \\
\hline $\mathrm{C}$ & 0.22285900 & 3.37828000 & 0.14892000 \\
\hline $\mathrm{C}$ & -4.10261900 & -1.62808500 & -0.23180300 \\
\hline $\mathrm{C}$ & -4.50410500 & -0.83024800 & -1.34653200 \\
\hline $\mathrm{C}$ & -5.15253400 & 0.39194400 & -1.12252100 \\
\hline $\mathrm{H}$ & -5.40013100 & 1.01568900 & -1.96756100 \\
\hline $\mathrm{C}$ & -5.46667700 & 0.86213200 & 0.14300000 \\
\hline $\mathrm{C}$ & -5.17390800 & 0.02319400 & 1.20974600 \\
\hline $\mathrm{H}$ & -5.46047500 & 0.36036400 & 2.19558100 \\
\hline $\mathrm{C}$ & -4.52633300 & -1.20643200 & 1.07036700 \\
\hline $\mathrm{C}$ & -4.32526900 & -2.02142200 & 2.38478400 \\
\hline $\mathrm{C}$ & -2.83753200 & -2.11320700 & 2.77867200 \\
\hline $\mathrm{H}$ & -2.38090400 & -1.11764000 & 2.79003200 \\
\hline $\mathrm{H}$ & -2.27428800 & -2.73012600 & 2.08086900 \\
\hline $\mathrm{H}$ & -2.74799700 & -2.54818800 & 3.78422700 \\
\hline $\mathrm{C}$ & -4.94684400 & -3.42585200 & 2.24928200 \\
\hline $\mathrm{H}$ & -4.82352400 & -3.97799400 & 3.19135800 \\
\hline $\mathrm{H}$ & -4.46339500 & -3.99060600 & 1.44952500 \\
\hline $\mathrm{H}$ & -6.02119000 & -3.34831200 & 2.03612000 \\
\hline $\mathrm{C}$ & -5.03671700 & -1.37255200 & 3.59345000 \\
\hline $\mathrm{H}$ & -6.11179300 & -1.23422600 & 3.42429700 \\
\hline $\mathrm{H}$ & -4.59616800 & -0.40743500 & 3.86862300 \\
\hline $\mathrm{H}$ & -4.92207700 & -2.03696200 & 4.45807300 \\
\hline $\mathrm{C}$ & -4.24243200 & -1.19821300 & -2.83699100 \\
\hline $\mathrm{C}$ & -4.83670100 & -2.58290300 & -3.16423700 \\
\hline
\end{tabular}




\begin{tabular}{|c|c|c|}
\hline-4.68523200 & -2.80969700 & -4.22885500 \\
\hline-5.91614000 & -2.59259600 & -2.96309100 \\
\hline-4.35750200 & -3.36616800 & -2.57341000 \\
\hline-2.73871700 & -1.14870300 & -3.17090700 \\
\hline-2.17629600 & -1.88807200 & -2.60191300 \\
\hline-2.32750400 & -0.15982300 & -2.93947100 \\
\hline-2.59184100 & -1.34204400 & -4.24292100 \\
\hline-4.92173300 & -0.21649800 & -3.81741500 \\
\hline-4.49347400 & 0.79079800 & -3.76352600 \\
\hline-6.00540600 & -0.14722000 & -3.65989500 \\
\hline-4.75849100 & -0.58079400 & -4.83867500 \\
\hline-6.19144000 & 2.19457300 & 0.38270200 \\
\hline-6.16917800 & 3.10072800 & -0.85814900 \\
\hline-6.65135700 & 4.05844700 & -0.62602100 \\
\hline-6.71098700 & 2.65533800 & -1.70000700 \\
\hline-5.14423100 & 3.30848600 & -1.18381100 \\
\hline-7.66080900 & 1.89251600 & 0.73792400 \\
\hline-8.21679600 & 2.82194100 & 0.92394200 \\
\hline-7.72442100 & 1.26930600 & 1.63691200 \\
\hline-8.15201000 & 1.35224100 & -0.07974400 \\
\hline-5.54682400 & 2.97355900 & 1.54525400 \\
\hline-4.48970100 & 3.17730300 & 1.35157300 \\
\hline-5.61311200 & 2.42709900 & 2.49127300 \\
\hline-6.06166100 & 3.93351200 & 1.68454400 \\
\hline 3.00038900 & 3.14765800 & 0.44444600 \\
\hline 1.37737500 & 2.46089600 & 0.23320200 \\
\hline 0.83587100 & 1.16596600 & 0.11503200 \\
\hline 1.42041400 & -0.13430700 & 0.13174700 \\
\hline 2.49186000 & -0.26511800 & 0.22382600 \\
\hline 0.61267000 & -1.23006400 & 0.03218700 \\
\hline 0.97071800 & -2.63428900 & 0.02459000 \\
\hline 2.19978500 & -3.26865500 & 0.16906300 \\
\hline 3.09687700 & -2.67331900 & 0.32787000 \\
\hline 2.25686900 & -4.65948900 & 0.11732300 \\
\hline 3.21418200 & -5.16703400 & 0.22458000 \\
\hline 1.08373200 & -5.40399600 & -0.06546000 \\
\hline 1.13868700 & -6.49080900 & -0.10224300 \\
\hline-0.15265200 & -4.77354400 & -0.19208700 \\
\hline-1.06061500 & -5.35865200 & -0.31811600 \\
\hline-0.22286200 & -3.37822200 & -0.14897500 \\
\hline 4.10259500 & 1.62808300 & 0.23184700 \\
\hline 4.50402900 & 0.83019700 & 1.34656900 \\
\hline 5.15240200 & -0.39202500 & 1.12253000 \\
\hline 5.39996300 & -1.01581400 & 1.96754800 \\
\hline
\end{tabular}




\begin{tabular}{|c|c|c|c|}
\hline $\mathrm{C}$ & 5.46657300 & -0.86217200 & -0.14299700 \\
\hline $\mathrm{C}$ & 5.17387800 & -0.02318500 & -1.20972200 \\
\hline $\mathrm{H}$ & 5.46047100 & -0.36032100 & -2.19556600 \\
\hline $\mathrm{C}$ & 4.52635100 & 1.20645500 & -1.07032300 \\
\hline $\mathrm{C}$ & 4.32549600 & 2.02148200 & -2.38474300 \\
\hline $\mathrm{C}$ & 2.83783600 & 2.11330500 & -2.77890000 \\
\hline $\mathrm{H}$ & 2.38117300 & 1.11775500 & -2.79030700 \\
\hline $\mathrm{H}$ & 2.27448700 & 2.73027200 & -2.08122600 \\
\hline $\mathrm{H}$ & 2.74850700 & 2.54824900 & -3.78448800 \\
\hline $\mathrm{C}$ & 4.94710800 & 3.42589000 & -2.24916500 \\
\hline $\mathrm{H}$ & 4.82396600 & 3.97801600 & -3.19127300 \\
\hline $\mathrm{H}$ & 4.46355300 & 3.99068600 & -1.44950100 \\
\hline $\mathrm{H}$ & 6.02141700 & 3.34830600 & -2.03583000 \\
\hline $\mathrm{C}$ & 5.03712500 & 1.37256100 & -3.59326900 \\
\hline $\mathrm{H}$ & 6.11218700 & 1.23430300 & -3.42396600 \\
\hline $\mathrm{H}$ & 4.59666500 & 0.40739400 & -3.86841400 \\
\hline $\mathrm{H}$ & 4.92256100 & 2.03690000 & -4.45795700 \\
\hline $\mathrm{C}$ & 4.24235000 & 1.19812000 & 2.83704900 \\
\hline $\mathrm{C}$ & 4.83654100 & 2.58283100 & 3.16433100 \\
\hline $\mathrm{H}$ & 4.68511900 & 2.80955500 & 4.22897100 \\
\hline $\mathrm{H}$ & 5.91596800 & 2.59261300 & 2.96312500 \\
\hline $\mathrm{H}$ & 4.35725100 & 3.36609700 & 2.57358000 \\
\hline $\mathrm{C}$ & 2.73865100 & 1.14848900 & 3.17103000 \\
\hline $\mathrm{H}$ & 2.17616000 & 1.88786000 & 2.60210800 \\
\hline $\mathrm{H}$ & 2.32748600 & 0.15959700 & 2.93955800 \\
\hline $\mathrm{H}$ & 2.59181200 & 1.34175600 & 4.24306400 \\
\hline $\mathrm{C}$ & 4.92173400 & 0.21645000 & 3.81745300 \\
\hline $\mathrm{H}$ & 4.49362000 & -0.79090300 & 3.76346600 \\
\hline $\mathrm{H}$ & 6.00542800 & 0.14734300 & 3.66000200 \\
\hline $\mathrm{H}$ & 4.75836800 & 0.58065700 & 4.83872600 \\
\hline $\mathrm{C}$ & 6.19129800 & -2.19462300 & -0.38271900 \\
\hline $\mathrm{C}$ & 6.16894800 & -3.10079600 & 0.85811400 \\
\hline $\mathrm{H}$ & 6.65117400 & -4.05849600 & 0.62600900 \\
\hline $\mathrm{H}$ & 6.71066900 & -2.65540300 & 1.70002600 \\
\hline $\mathrm{H}$ & 5.14397800 & -3.30858800 & 1.18368400 \\
\hline $\mathrm{C}$ & 7.66069500 & -1.89261300 & -0.73787400 \\
\hline $\mathrm{H}$ & 8.21665600 & -2.82205200 & -0.92390400 \\
\hline $\mathrm{H}$ & 7.72437300 & -1.26936800 & -1.63683300 \\
\hline $\mathrm{H}$ & 8.15188100 & -1.35238900 & 0.07983700 \\
\hline $\mathrm{C}$ & 5.54667600 & -2.97353500 & -1.54532100 \\
\hline $\mathrm{H}$ & 4.48955400 & -3.17729300 & -1.35164700 \\
\hline $\mathrm{H}$ & 5.61295400 & -2.42699600 & -2.49129700 \\
\hline $\mathrm{H}$ & 6.06151100 & -3.93347700 & -1.68468800 \\
\hline
\end{tabular}


Table S6. Cartesian coordinates $(x, y, z)$ for the optimized structure of $\mathbf{1}^{2 \cdots-*}$ featuring an open-shell singlet state.

\begin{tabular}{|c|c|c|c|}
\hline $\mathrm{P}$ & -3.01083900 & -3.14297000 & -0.43441200 \\
\hline $\mathrm{C}$ & -1.38470000 & -2.48940700 & -0.23173200 \\
\hline $\mathrm{C}$ & -0.83362400 & -1.15750800 & -0.11679100 \\
\hline $\mathrm{C}$ & -1.41198100 & 0.12378800 & -0.13906100 \\
\hline $\mathrm{H}$ & -2.48156300 & 0.26094800 & -0.23899400 \\
\hline $\mathrm{C}$ & -0.59367300 & 1.23547700 & -0.03298900 \\
\hline $\mathrm{C}$ & -0.95169800 & 2.63805100 & -0.03183400 \\
\hline $\mathrm{C}$ & -2.18374000 & 3.26532400 & -0.18097900 \\
\hline $\mathrm{H}$ & -3.07604900 & 2.66157300 & -0.33818600 \\
\hline $\mathrm{C}$ & -2.25581600 & 4.65574300 & -0.13695000 \\
\hline $\mathrm{H}$ & -3.21643000 & 5.15602200 & -0.24838200 \\
\hline $\mathrm{C}$ & -1.08440700 & 5.40838600 & 0.04465700 \\
\hline $\mathrm{H}$ & -1.14721900 & 6.49547400 & 0.07632600 \\
\hline $\mathrm{C}$ & 0.15515300 & 4.79201100 & 0.17720500 \\
\hline $\mathrm{H}$ & 1.05445800 & 5.39106200 & 0.30352800 \\
\hline $\mathrm{C}$ & 0.24508000 & 3.39073600 & 0.14151300 \\
\hline $\mathrm{C}$ & -4.10547000 & -1.62350600 & -0.21958700 \\
\hline $\mathrm{C}$ & -4.51116500 & -0.83475800 & -1.33747400 \\
\hline $\mathrm{C}$ & -5.15333500 & 0.39067300 & -1.11621700 \\
\hline $\mathrm{H}$ & -5.40421300 & 1.01003600 & -1.96342600 \\
\hline $\mathrm{C}$ & -5.45520000 & 0.86997300 & 0.14904500 \\
\hline $\mathrm{C}$ & -5.15845400 & 0.03662800 & 1.21921400 \\
\hline $\mathrm{H}$ & -5.43542400 & 0.38111100 & 2.20513600 \\
\hline $\mathrm{C}$ & -4.51570800 & -1.19554200 & 1.08306100 \\
\hline $\mathrm{C}$ & -4.30329500 & -2.00553700 & 2.39805800 \\
\hline $\mathrm{C}$ & -2.81241100 & -2.09287500 & 2.78221300 \\
\hline $\mathrm{H}$ & -2.35905700 & -1.09579400 & 2.78645900 \\
\hline $\mathrm{H}$ & -2.24438300 & -2.70746900 & 2.08592900 \\
\hline $\mathrm{H}$ & -2.71591100 & -2.52374100 & 3.78874100 \\
\hline $\mathrm{C}$ & -4.92462800 & -3.41110900 & 2.27294700 \\
\hline $\mathrm{H}$ & -4.79190300 & -3.96007500 & 3.21533600 \\
\hline $\mathrm{H}$ & -4.45129600 & -3.98204600 & 1.47176800 \\
\hline $\mathrm{H}$ & -6.00081700 & -3.33559200 & 2.06906700 \\
\hline $\mathrm{C}$ & -5.00772200 & -1.35241800 & 3.60834800 \\
\hline $\mathrm{H}$ & -6.08359800 & -1.21462500 & 3.44426200 \\
\hline $\mathrm{H}$ & -4.56549000 & -0.38626000 & 3.87638800 \\
\hline $\mathrm{H}$ & -4.88795000 & -2.01301100 & 4.47513200 \\
\hline $\mathrm{C}$ & -4.26123300 & -1.21834900 & -2.82569900 \\
\hline $\mathrm{C}$ & -4.86534200 & -2.60310600 & -3.13495200 \\
\hline $\mathrm{H}$ & -4.71912600 & -2.84305600 & -4.19718400 \\
\hline $\mathrm{H}$ & -5.94387800 & -2.60393600 & -2.92949000 \\
\hline
\end{tabular}




\begin{tabular}{|c|c|c|c|}
\hline $\mathrm{H}$ & -4.39149000 & -3.38560700 & -2.53898700 \\
\hline $\mathrm{C}$ & -2.75993700 & -1.17904400 & -3.17331700 \\
\hline $\mathrm{H}$ & -2.19037100 & -1.91714300 & -2.60946700 \\
\hline $\mathrm{H}$ & -2.34085700 & -0.19270000 & -2.94666600 \\
\hline $\mathrm{H}$ & -2.62452100 & -1.37549300 & -4.24615800 \\
\hline $\mathrm{C}$ & -4.94412100 & -0.24264000 & -3.80927900 \\
\hline $\mathrm{H}$ & -4.51081700 & 0.76286600 & -3.76713600 \\
\hline $\mathrm{H}$ & -6.02599500 & -0.16709200 & -3.64318700 \\
\hline $\mathrm{H}$ & -4.79097800 & -0.61650900 & -4.82861500 \\
\hline $\mathrm{C}$ & -6.17038000 & 2.20821500 & 0.38521500 \\
\hline $\mathrm{C}$ & -6.14485300 & 3.10905700 & -0.85948800 \\
\hline $\mathrm{H}$ & -6.61910400 & 4.07112500 & -0.62936600 \\
\hline $\mathrm{H}$ & -6.69307100 & 2.66505900 & -1.69803400 \\
\hline $\mathrm{H}$ & -5.11920800 & 3.30809600 & -1.18816500 \\
\hline $\mathrm{C}$ & -7.64062400 & 1.91627100 & 0.74533200 \\
\hline $\mathrm{H}$ & -8.19038400 & 2.84995500 & 0.92803200 \\
\hline $\mathrm{H}$ & -7.70583300 & 1.29814200 & 1.64778200 \\
\hline $\mathrm{H}$ & -8.13734700 & 1.37498300 & -0.06840400 \\
\hline $\mathrm{C}$ & -5.51748900 & 2.98816500 & 1.54250900 \\
\hline $\mathrm{H}$ & -4.46009300 & 3.18619900 & 1.34435200 \\
\hline $\mathrm{H}$ & -5.58456700 & 2.44661900 & 2.49136100 \\
\hline $\mathrm{H}$ & -6.02715600 & 3.95123200 & 1.67878900 \\
\hline $\mathrm{P}$ & 3.01085000 & 3.14292100 & 0.43456700 \\
\hline $\mathrm{C}$ & 1.38472700 & 2.48937300 & 0.23170900 \\
\hline $\mathrm{C}$ & 0.83365900 & 1.15747500 & 0.11670500 \\
\hline $\mathrm{C}$ & 1.41201700 & -0.12382200 & 0.13894100 \\
\hline $\mathrm{H}$ & 2.48160400 & -0.26098300 & 0.23882100 \\
\hline $\mathrm{C}$ & 0.59371100 & -1.23550900 & 0.03287500 \\
\hline $\mathrm{C}$ & 0.95172500 & -2.63808600 & 0.03181300 \\
\hline $\mathrm{C}$ & 2.18375300 & -3.26536400 & 0.18104700 \\
\hline $\mathrm{H}$ & 3.07607600 & -2.66162500 & 0.33823700 \\
\hline $\mathrm{C}$ & 2.25580800 & -4.65578700 & 0.13713500 \\
\hline $\mathrm{H}$ & 3.21641400 & -5.15607000 & 0.24861500 \\
\hline $\mathrm{C}$ & 1.08439300 & -5.40843300 & -0.04441600 \\
\hline $\mathrm{H}$ & 1.14719700 & -6.49552500 & -0.07599900 \\
\hline $\mathrm{C}$ & -0.15515700 & -4.79205600 & -0.17702700 \\
\hline $\mathrm{H}$ & -1.05447000 & -5.39110300 & -0.30330800 \\
\hline $\mathrm{C}$ & -0.24506200 & -3.39077400 & -0.14146700 \\
\hline $\mathrm{C}$ & 4.10550000 & 1.62349100 & 0.21965200 \\
\hline $\mathrm{C}$ & 4.51108600 & 0.83460400 & 1.33747100 \\
\hline $\mathrm{C}$ & 5.15327000 & -0.39080400 & 1.11611900 \\
\hline $\mathrm{H}$ & 5.40411300 & -1.01027300 & 1.96326200 \\
\hline $\mathrm{C}$ & 5.45522100 & -0.86995600 & -0.14917500 \\
\hline $\mathrm{C}$ & 5.15857700 & -0.03646800 & -1.21926700 \\
\hline
\end{tabular}

\section{S18}




\begin{tabular}{|c|c|c|c|}
\hline $\mathrm{H}$ & 5.43566200 & -0.38082100 & -2.20520300 \\
\hline $\mathrm{C}$ & 4.51582100 & 1.19569000 & -1.08302300 \\
\hline $\mathrm{C}$ & 4.30342500 & 2.00586600 & -2.39792700 \\
\hline $\mathrm{C}$ & 2.81255000 & 2.09323200 & -2.78209200 \\
\hline $\mathrm{H}$ & 2.35921600 & 1.09614100 & -2.78647600 \\
\hline $\mathrm{H}$ & 2.24450500 & 2.70772800 & -2.08573700 \\
\hline $\mathrm{H}$ & 2.71606500 & 2.52422300 & -3.78856700 \\
\hline $\mathrm{C}$ & 4.92472100 & 3.41143600 & -2.27260800 \\
\hline $\mathrm{H}$ & 4.79202100 & 3.96052100 & -3.21493100 \\
\hline $\mathrm{H}$ & 4.45134700 & 3.98225900 & -1.47137600 \\
\hline $\mathrm{H}$ & 6.00090300 & 3.33590500 & -2.06870200 \\
\hline $\mathrm{C}$ & 5.00786700 & 1.35299800 & -3.60834200 \\
\hline $\mathrm{H}$ & 6.08374500 & 1.21519800 & -3.44427900 \\
\hline $\mathrm{H}$ & 4.56565100 & 0.38689200 & -3.87659400 \\
\hline $\mathrm{H}$ & 4.88808600 & 2.01377800 & -4.47498400 \\
\hline $\mathrm{C}$ & 4.26102900 & 1.21802900 & 2.82571000 \\
\hline $\mathrm{C}$ & 4.86494600 & 2.60283800 & 3.13510700 \\
\hline $\mathrm{H}$ & 4.71866400 & 2.84267300 & 4.19735800 \\
\hline $\mathrm{H}$ & 5.94348800 & 2.60383100 & 2.92968100 \\
\hline $\mathrm{H}$ & 4.39100700 & 3.38533200 & 2.53919800 \\
\hline $\mathrm{C}$ & 2.75971900 & 1.17852100 & 3.17324600 \\
\hline $\mathrm{H}$ & 2.19008200 & 1.91653600 & 2.60935800 \\
\hline $\mathrm{H}$ & 2.34078400 & 0.19211900 & 2.94658700 \\
\hline $\mathrm{H}$ & 2.62422000 & 1.37496900 & 4.24607400 \\
\hline $\mathrm{C}$ & 4.94398500 & 0.24231400 & 3.80924000 \\
\hline $\mathrm{H}$ & 4.51076700 & -0.76322400 & 3.76702400 \\
\hline $\mathrm{H}$ & 6.02587000 & 0.16686600 & 3.64316500 \\
\hline $\mathrm{H}$ & 4.79079500 & 0.61610600 & 4.82859700 \\
\hline $\mathrm{C}$ & 6.17042600 & -2.20817200 & -0.38546300 \\
\hline $\mathrm{C}$ & 6.14515000 & -3.10901100 & 0.85924500 \\
\hline $\mathrm{H}$ & 6.61939200 & -4.07106300 & 0.62904100 \\
\hline $\mathrm{H}$ & 6.69350100 & -2.66499000 & 1.69769200 \\
\hline $\mathrm{H}$ & 5.11957600 & -3.30809900 & 1.18811700 \\
\hline $\mathrm{C}$ & 7.64059900 & -1.91619100 & -0.74580800 \\
\hline $\mathrm{H}$ & 8.19034800 & -2.84986400 & -0.92859700 \\
\hline $\mathrm{H}$ & 7.70568000 & -1.29805600 & -1.64826300 \\
\hline $\mathrm{H}$ & 8.13743000 & -1.37489900 & 0.06786100 \\
\hline $\mathrm{C}$ & 5.51737500 & -2.98814900 & -1.54265200 \\
\hline $\mathrm{H}$ & 4.46003700 & -3.18629800 & -1.34429500 \\
\hline $\mathrm{H}$ & 5.58419700 & -2.44656100 & -2.49149900 \\
\hline $\mathrm{H}$ & 6.02711300 & -3.95116100 & -1.67906100 \\
\hline
\end{tabular}


Table S7. Cartesian coordinates $(x, y, z)$ for the optimized structure of $\mathbf{1}^{2-\cdots}$ featuring a triplet state.

\begin{tabular}{|c|c|c|c|}
\hline $\mathrm{P}$ & -3.01368200 & -3.13787900 & -0.45114900 \\
\hline $\mathrm{C}$ & -1.38429000 & -2.50257300 & -0.23877600 \\
\hline $\mathrm{C}$ & -0.83085000 & -1.15440000 & -0.12087600 \\
\hline $\mathrm{C}$ & -1.40846400 & 0.12004000 & -0.14726200 \\
\hline $\mathrm{H}$ & -2.47697600 & 0.25834000 & -0.25421200 \\
\hline $\mathrm{C}$ & -0.58787800 & 1.23795600 & -0.03549200 \\
\hline $\mathrm{C}$ & -0.94591300 & 2.64099300 & -0.03757400 \\
\hline $\mathrm{C}$ & -2.17756400 & 3.26519000 & -0.19369500 \\
\hline $\mathrm{H}$ & -3.06737100 & 2.65814600 & -0.35436200 \\
\hline $\mathrm{C}$ & -2.25633200 & 4.65623100 & -0.15271100 \\
\hline $\mathrm{H}$ & -3.21771200 & 5.15368100 & -0.26947500 \\
\hline $\mathrm{C}$ & -1.08575200 & 5.41147700 & 0.03323700 \\
\hline $\mathrm{H}$ & -1.15157200 & 6.49875300 & 0.06248700 \\
\hline $\mathrm{C}$ & 0.15456600 & 4.80124500 & 0.17327300 \\
\hline $\mathrm{H}$ & 1.04973400 & 5.40610700 & 0.30305300 \\
\hline $\mathrm{C}$ & 0.25298400 & 3.39676700 & 0.14123700 \\
\hline $\mathrm{C}$ & -4.10429100 & -1.62033400 & -0.22206000 \\
\hline $\mathrm{C}$ & -4.51571800 & -0.82911100 & -1.33535000 \\
\hline $\mathrm{C}$ & -5.15429300 & 0.39644600 & -1.10598100 \\
\hline $\mathrm{H}$ & -5.41001600 & 1.01897900 & -1.94941600 \\
\hline $\mathrm{C}$ & -5.44494400 & 0.87293500 & 0.16305700 \\
\hline $\mathrm{C}$ & -5.14222700 & 0.03616400 & 1.22894800 \\
\hline $\mathrm{H}$ & -5.41021300 & 0.37876200 & 2.21802800 \\
\hline $\mathrm{C}$ & -4.50386000 & -1.19722200 & 1.08475100 \\
\hline $\mathrm{C}$ & -4.28542000 & -2.01444500 & 2.39401000 \\
\hline $\mathrm{C}$ & -2.79309300 & -2.10547800 & 2.77234200 \\
\hline $\mathrm{H}$ & -2.33767700 & -1.10934300 & 2.77477600 \\
\hline $\mathrm{H}$ & -2.22559300 & -2.71999600 & 2.07542100 \\
\hline $\mathrm{H}$ & -2.69428400 & -2.53654900 & 3.77848800 \\
\hline $\mathrm{C}$ & -4.90864300 & -3.41878200 & 2.26397500 \\
\hline $\mathrm{H}$ & -4.77058300 & -3.97362500 & 3.20200500 \\
\hline $\mathrm{H}$ & -4.44155100 & -3.98665800 & 1.45686200 \\
\hline $\mathrm{H}$ & -5.98588300 & -3.34142500 & 2.06664900 \\
\hline $\mathrm{C}$ & -4.98478000 & -1.36700400 & 3.61021900 \\
\hline $\mathrm{H}$ & -6.06068200 & -1.22553600 & 3.44969500 \\
\hline $\mathrm{H}$ & -4.53932300 & -0.40357800 & 3.88247300 \\
\hline $\mathrm{H}$ & -4.86420500 & -2.03300300 & 4.47271900 \\
\hline $\mathrm{C}$ & -4.27531200 & -1.21062300 & -2.82549900 \\
\hline $\mathrm{C}$ & -4.88514800 & -2.59324500 & -3.13345900 \\
\hline $\mathrm{H}$ & -4.74441200 & -2.83263300 & -4.19647100 \\
\hline $\mathrm{H}$ & -5.96264500 & -2.59112900 & -2.92294300 \\
\hline
\end{tabular}




\begin{tabular}{|c|c|c|}
\hline-4.41191100 & -3.37916700 & -2.54136500 \\
\hline-2.77606400 & -1.17477800 & -3.18321700 \\
\hline-2.20264200 & -1.91720200 & -2.62894000 \\
\hline-2.35169000 & -0.19164100 & -2.95307400 \\
\hline-2.64901400 & -1.36521200 & -4.25811900 \\
\hline-4.96184400 & -0.23101000 & -3.80244100 \\
\hline-4.52585100 & 0.77324300 & -3.75995600 \\
\hline-6.04241800 & -0.15335500 & -3.62934200 \\
\hline-4.81589300 & -0.60246300 & -4.82369300 \\
\hline-6.15432100 & 2.21263600 & 0.40816400 \\
\hline-6.13883100 & 3.11513800 & -0.83545900 \\
\hline-6.60814300 & 4.07802900 & -0.59892500 \\
\hline-6.69701200 & 2.67399900 & -1.66901200 \\
\hline-5.11608100 & 3.31229800 & -1.17401700 \\
\hline-7.62165700 & 1.92346400 & 0.78219500 \\
\hline-8.16749500 & 2.85827300 & 0.97057800 \\
\hline-7.67978700 & 1.30512300 & 1.68502700 \\
\hline-8.12746100 & 1.38367700 & -0.02698800 \\
\hline-5.48767600 & 2.98915100 & 1.55986400 \\
\hline-4.43265000 & 3.18783600 & 1.34982300 \\
\hline-5.54420900 & 2.44517000 & 2.50805300 \\
\hline-5.99563000 & 3.95191500 & 1.70427400 \\
\hline 3.01383300 & 3.13789000 & 0.45124300 \\
\hline 1.38443000 & 2.50262300 & 0.23884400 \\
\hline 0.83097700 & 1.15445700 & 0.12095100 \\
\hline 1.40859000 & -0.11998200 & 0.14733900 \\
\hline 2.47709900 & -0.25828200 & 0.25432600 \\
\hline 0.58800600 & -1.23789600 & 0.03555900 \\
\hline 0.94605000 & -2.64093000 & 0.03766200 \\
\hline 2.17770600 & -3.26511400 & 0.19379200 \\
\hline 3.06750200 & -2.65805600 & 0.35445300 \\
\hline 2.25648400 & -4.65615500 & 0.15282600 \\
\hline 3.21786400 & -5.15360000 & 0.26960700 \\
\hline 1.08590800 & -5.41140900 & -0.03311600 \\
\hline 1.15173400 & -6.49868600 & -0.06235300 \\
\hline-0.15441300 & -4.80118700 & -0.17316700 \\
\hline-1.04957700 & -5.40605500 & -0.30294400 \\
\hline-0.25284100 & -3.39670900 & -0.14114600 \\
\hline 4.10435500 & 1.62030200 & 0.22207600 \\
\hline 4.51562900 & 0.82892100 & 1.33530500 \\
\hline 5.15414700 & -0.39665100 & 1.10583800 \\
\hline 5.40977200 & -1.01930400 & 1.94921300 \\
\hline 5.44484000 & -0.87301700 & -0.16323800 \\
\hline 5.14224100 & -0.03610600 & -1.22905700 \\
\hline
\end{tabular}




\begin{tabular}{|c|c|c|c|}
\hline $\mathrm{H}$ & 5.41024500 & -0.37861300 & -2.21815800 \\
\hline $\mathrm{C}$ & 4.50397100 & 1.19731800 & -1.08475400 \\
\hline $\mathrm{C}$ & 4.28561300 & 2.01474600 & -2.39390000 \\
\hline $\mathrm{C}$ & 2.79326100 & 2.10622200 & -2.77203000 \\
\hline $\mathrm{H}$ & 2.33758500 & 1.11020600 & -2.77454900 \\
\hline $\mathrm{H}$ & 2.22600800 & 2.72078000 & -2.07493900 \\
\hline $\mathrm{H}$ & 2.69443000 & 2.53747100 & -3.77809600 \\
\hline $\mathrm{C}$ & 4.90919200 & 3.41890900 & -2.26372600 \\
\hline $\mathrm{H}$ & 4.77113400 & 3.97394000 & -3.20164500 \\
\hline $\mathrm{H}$ & 4.44235200 & 3.98676200 & -1.45644900 \\
\hline $\mathrm{H}$ & 5.98644200 & 3.34126000 & -2.06656400 \\
\hline $\mathrm{C}$ & 4.98466800 & 1.36730600 & -3.61028900 \\
\hline $\mathrm{H}$ & 6.06051900 & 1.22536300 & -3.44984100 \\
\hline $\mathrm{H}$ & 4.53880700 & 0.40413000 & -3.88276400 \\
\hline $\mathrm{H}$ & 4.86433100 & 2.03355800 & -4.47262600 \\
\hline $\mathrm{C}$ & 4.27506300 & 1.21031800 & 2.82546200 \\
\hline $\mathrm{C}$ & 4.88484200 & 2.59293200 & 3.13355100 \\
\hline $\mathrm{H}$ & 4.74392600 & 2.83230500 & 4.19654300 \\
\hline $\mathrm{H}$ & 5.96237300 & 2.59082200 & 2.92321500 \\
\hline $\mathrm{H}$ & 4.41170900 & 3.37886500 & 2.54138800 \\
\hline $\mathrm{C}$ & 2.77576600 & 1.17447900 & 3.18298400 \\
\hline $\mathrm{H}$ & 2.20242900 & 1.91692700 & 2.62864500 \\
\hline $\mathrm{H}$ & 2.35140900 & 0.19135200 & 2.95277100 \\
\hline $\mathrm{H}$ & 2.64857700 & 1.36489200 & 4.25787300 \\
\hline $\mathrm{C}$ & 4.96146900 & 0.23067000 & 3.80246000 \\
\hline $\mathrm{H}$ & 4.52553900 & -0.77360400 & 3.75982000 \\
\hline $\mathrm{H}$ & 6.04208000 & 0.15309000 & 3.62956600 \\
\hline $\mathrm{H}$ & 4.81530300 & 0.60203600 & 4.82371300 \\
\hline $\mathrm{C}$ & 6.15414400 & -2.21274600 & -0.40841000 \\
\hline $\mathrm{C}$ & 6.13875000 & -3.11519200 & 0.83524800 \\
\hline $\mathrm{H}$ & 6.60803500 & -4.07809800 & 0.59871600 \\
\hline $\mathrm{H}$ & 6.69700600 & -2.67401700 & 1.66873200 \\
\hline $\mathrm{H}$ & 5.11602900 & -3.31232600 & 1.17390500 \\
\hline $\mathrm{C}$ & 7.62145900 & -1.92363900 & -0.78258900 \\
\hline $\mathrm{H}$ & 8.16724500 & -2.85847100 & -0.97101200 \\
\hline $\mathrm{H}$ & 7.67952100 & -1.30531100 & -1.68543400 \\
\hline $\mathrm{H}$ & 8.12736200 & -1.38385800 & 0.02653600 \\
\hline $\mathrm{C}$ & 5.48736300 & -2.98930600 & -1.56000700 \\
\hline $\mathrm{H}$ & 4.43232200 & -3.18784700 & -1.34990800 \\
\hline $\mathrm{H}$ & 5.54392100 & -2.44543700 & -2.50825800 \\
\hline $\mathrm{H}$ & 5.99519800 & -3.95214300 & -1.70434000 \\
\hline
\end{tabular}




\section{References}

(1) Cowley, A. H.; Norman, N. C.; Pakulski, M.; Becker, G.; Layh, M.; Kirchner, E.; Schmidt, M., Phosphorus Compounds Containing Sterically Demanding Groups. In Inorganic Syntheses, John Wiley \& Sons, Inc.: 2007; pp 235-240.

(2) Merlet, S.; Birau, M.; Wang, Z. Y., Org. Lett. 2002, 4, 2157-2159.

(3) Gaussian 09, Revision B.01, Frisch, M. J.; Trucks, G. W.; Schlegel, H. B.; Scuseria, G. E.; Robb, M. A.; Cheeseman, J. R.; Scalmani, G.; Barone, V.; Mennucci, B.; Petersson, G. A.; Nakatsuji, H.; Caricato, M.; Li, X.; Hratchian, H. P.; Izmaylov, A. F.; Bloino, J.; Zheng, G.; Sonnenberg, J. L.; Hada, M.; Ehara, M.; Toyota, K.; Fukuda, R.; Hasegawa, J.; Ishida, M.; Nakajima, T.; Honda, Y.; Kitao, O.; Nakai, H.; Vreven, T.; Montgomery, Jr., J. A.; Peralta, J. E.; Ogliaro, F.; Bearpark, M.; Heyd, J. J.; Brothers, E.; Kudin, K. N.; Staroverov, V. N.; Kieth, T.; Kobayashi, R.; Normand, J.; Raghavachari, K.; Rendell, A.; Burant, J. C.; Iyengar, S. S.; Tomasi, J.; Cossi, M.; Rega, N.; Millam, N. J.; Klene, M.; Knox, J. E.; Cross, J. B.; Bakken, V.; Adamo, C.; Jaramillo, J.; Gomperts, R.; Stratmann, R. E.; Yazyev, O.; Austin, A. J.; Cammi, R.; Pomelli, C.; Ochterski, J. W.; Martin, R. L.; Morokuma, K.; Zakrzewski, V. G.; Voth, G. A.; Salvador, P.; Dannenberg, J. J.; Dapprich, S.; Daniels, A. D.; Farkas, Ö.; Foresman, J. B.; Ortiz, J. V.; Cioslowski, J.; and Fox, D. J., Gaussian, Inc., Wallingford CT, 2010. 